Research Paper

\title{
Comparative analysis of Notch1 and Notch2 binding sites in the genome of BxPC3 pancreatic cancer cells
}

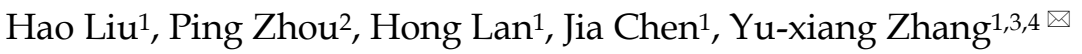 \\ 1. Department of Biochemistry and Molecular Biology, School of Basic Medical Sciences, Capital Medical University, Fengtai District, Beijing, 100069 China. \\ 2. Department of Bioinformatics and Computer Science, School of Biomedical Engineering, Capital Medical University, Beijing, China. \\ 3. Cancer Institute of Capital Medical University, Beijing, China. \\ 4. Beijing Key Laboratory for Cancer Invasion and Metastasis Research, Capital Medical University, Beijing, China. \\ $\square$ Corresponding author: Prof. Yu-xiang Zhang, Department of Biochemistry and Molecular Biology, School of Basic Medical Sciences, Capital Medical \\ University, Beijing, China. Tel: 86-10-83950122; E-mail: yxzhang@ccmu.edu.cn.
}

(C) Ivyspring International Publisher. This is an open access article distributed under the terms of the Creative Commons Attribution (CC BY-NC) license (https://creativecommons.org/licenses/by-nc/4.0/). See http://ivyspring.com/terms for full terms and conditions.

Received: 2016.07.05; Accepted: 2016.09.26; Published: 2017.01.01

\begin{abstract}
Notch signaling plays a key role in the development of pancreatic cancer. Among the four identified Notch receptors, Notch 1 and Notch2 share the highest homology. Notch1 has been reported to be an oncogene but some reports indicate that Notch2, not Notch 1, plays a key role in pancreatic carcinogenesis. As both are transcription factors, examination of their genomic binding sites might reveal interesting functional differences between them. Notch proteins do not have DNA-binding domain. In the canonical Notch signaling pathway, ligand binding induces the release and nuclear translocation of Notch receptor intracellular domains (NICDs), which then interact with the transcription factor CSL, resulting in subsequent activation of the canonical Notch target genes. We investigated the binding site profiles of Notchland Notch2 in the BxPC3 genome using CHIP-Seq and bioinfomatics. We found that Notch 1, Notch2 and CSL generally bound to different target genes. We also found that only a small subset of Notch 1 and Notch2 binding sites overlap with that of CSL, but about half of the CSL binding overlap with that of Notch1 or Notch2, indicating most Notch signaling activities are CSL-independent.
\end{abstract}

Key words: Notch, Genome binding sites, Pancreatic Cancer.

\section{Introduction}

Aberrantly activated Notch signaling plays key roles in carcinogenesis and the progression of human malignancies [1]. However, Notch signaling has been reported to play either oncogenic or tumor suppressor roles depending on the tissue context [2]. The Notch signaling network is frequently deregulated in pancreatic cancer, with up-regulated expression of Notch receptors and their ligands [3]. To date, four Notch receptors have been identified in mammals [4] and the presence of multiple Notch receptors and ligands raises the question whether different receptors play different roles in the same cell type. Of the four Notch receptors, Notch1 and Notch2 have the highest homology in both extracellular and intracellular domains (Fig. 1A). Nevertheless they have opposite effects on the growth of embryonic brain tumors growth, suggesting that they play opposing roles in a single tumor type [5].

Although Notch1 has been reported to be oncogenic for pancreatic cancer, there is evidence that Notch2, rather than Notch1 plays a key role in pancreatic carcinogenesis [6, 7]. Therefore, it is important to investigate the functional differences between Notch1 and Notch2 in pancreatic cancer cells. Notch receptors do not have DNA binding domains, so it is impossible to searching their targets based on genome sequences. In the canonical Notch signaling pathway, ligand binding induces a series of cleavages of the full-length receptors, and Notch receptor intracellular domains (NICDs) then translocate to the nucleus, interact with the transcription factor CSL (CBF1, Suppressor of 
Hairless, Lag1) which is then converted from a transcriptional repressor to a transcriptional activator [8] (Fig. 1B). As Notch1 and Notch2 are transcription factors, a closer examination of Notch1 and Notch2 genome binding data may reveal interesting functional differences in these Notch paralogues. CHIP-Seq was used for the identification of Notch1, Notch 2 and CSL binding sites. Fig. 2 provides a schematic outline of the experimental procedure.

\section{Materials and Methods}

\section{Cell culture}

Human pancreatic BxPC3 cells were obtained from the ATCC (Manassas, VA) and maintained in Dulbecco's modified Eagle's medium (DMEM) containing 10\% fetal bovine serum (Life Technologies, Gaithersburg, MD). Cells were incubated in $75 \mathrm{~cm}^{2}$ culture flasks in a humidified atmosphere at $37^{\circ} \mathrm{C}$ with $5 \%$ carbon dioxide in air, and passaged on reaching $80 \%$ confluence.
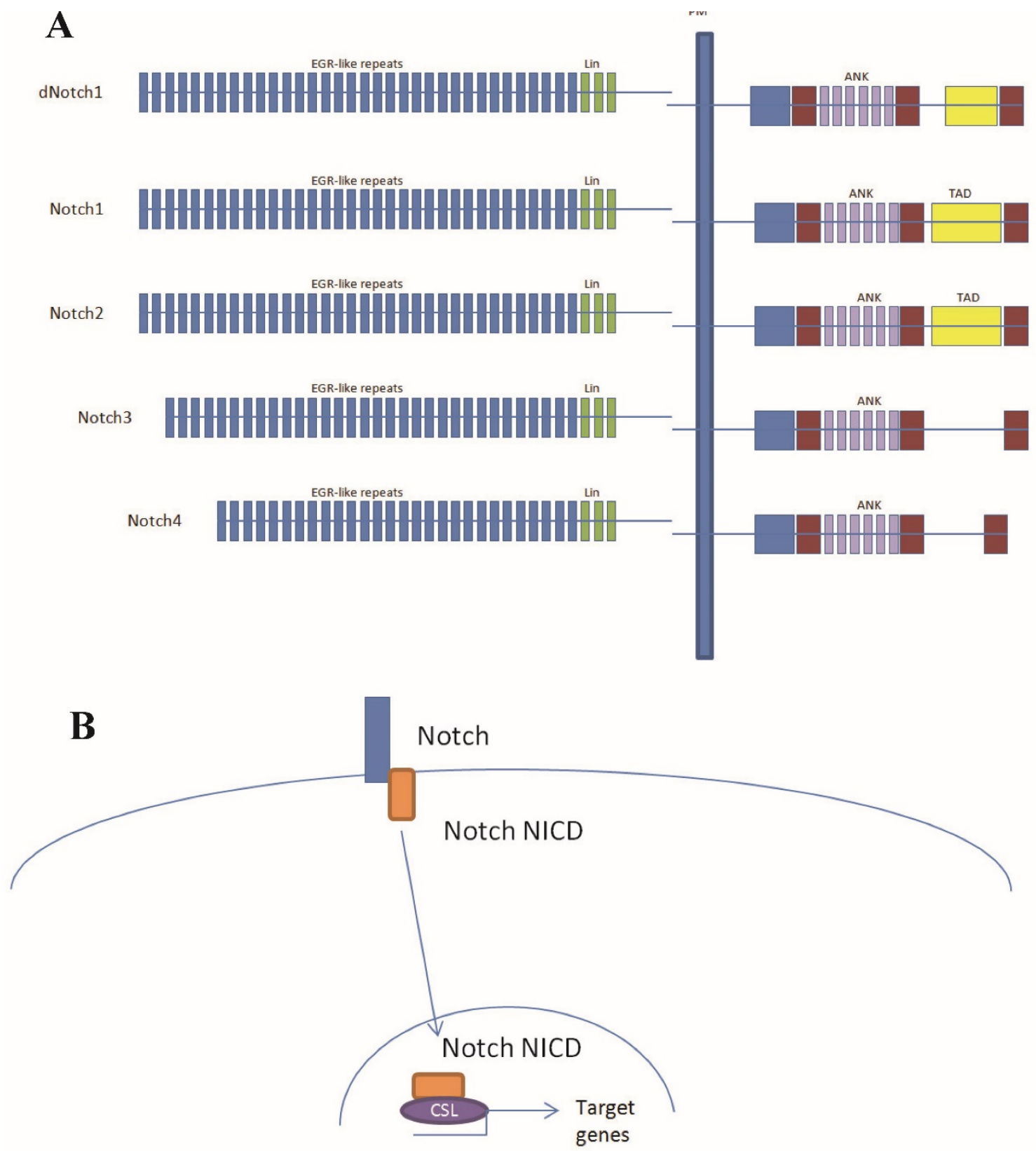

Figure 1. Notch protein domains and the canonical Notch signal transduction pathway. (A) Diagrammatic representation of the domains of the Drosophila Notch (dNotch) receptor and the four known mammalian receptors. Note that Notch3 and Notch4 contain no transcriptional activation domain TAD domain. PM, plasma membrane; EGFLR, epidermal growth-factor-like repeats; ANK, Ankyrin/CDC10 repeats; NLS, nuclear localization signals; TAD, transcriptional activation domain; PEST, Pro Glu Ser Thr (PEST) sequence. (B) Notch does not directly bind to DNA. In the canonical Notch signal transduction model, Notch does not directly bind to DNA, rather goes through CSL. In the absence of Notch intracellular domain (NICD), CSL binds to co-repressors. The interaction CSL with ANK repeats of the NICD displace these repressors to generate a transcriptional activator complex. 


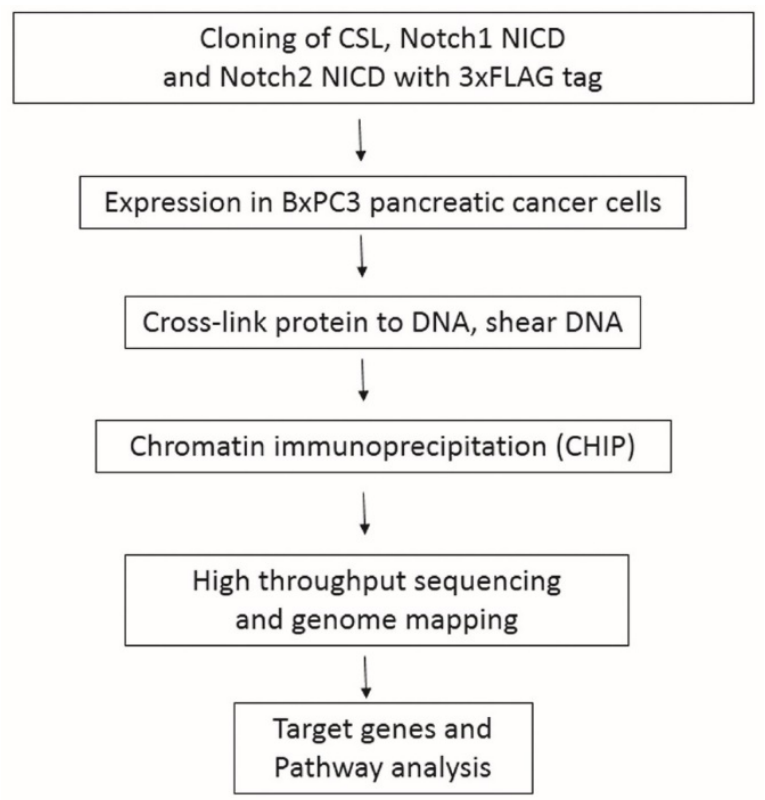

Figure 2. A schematic diagram of the experimental procedures.

\section{Plasmid constructions}

BxPC 3 cells were grown on plates and total RNA was isolated using Trizol reagent (Life Technologies, Gaithersburg, MD), followed by clean up with an RNeasy® Mini kit (Qiagen, Valencia, CA). RNA was reverse-transcribed to cDNA. Notch1, Notch2 intracellular segments and CSL cDNA were amplified and cloned into pcDNA3.0-3×Flag. The ligation products were transformed into competent cells; transformants were cultured on solid LB medium overnight and suitable clones selected.

\section{Western blotting}

Cells were seeded at $1 \times 10^{6}$ per well in $5 \mathrm{ml}$ of culture medium in $75 \mathrm{~cm}^{2}$ culture flasks, and allowed to grow overnight. SDS lysis buffer $(0.05 \mathrm{mM}$ Tris- $\mathrm{HCl}, 50 \mathrm{mM}$ BME, 2\% SDS, 0.1\% Bromophenol blue, $10 \%$ glycerol) was used to lyse the cells. Heat denatured proteins were then loaded, separated on an SDS-page gel, transferred onto a pure nitrocellulose membrane (Bio-Rad), and blocked with 5\% milk. Membranes were incubated with anti-Flag antibody (1:500 dilution; M2, Sigma-Aldrich, St. Louis, MO) or mouse anti-human $\beta$-actin monoclonal antibody (1:100 dilution; Santa Cruz Biotechnology; Santa Cruz, CA) overnight at $4^{\circ} \mathrm{C}$, then washed four times with TBST at room temperature for $5 \mathrm{~min}$ each wash, and incubated with horseradish peroxidase-conjugated rabbit antimouse immunoglobulin G (1:5000 dilution; Zymed, San Francisco, CA) for $60 \mathrm{~min}$ at room temperature. The membranes were washed again four times with TBST at room temperature $(5 \mathrm{~min}$ each wash) and protein bands were visualized by enhanced chemiluminescence (ECL Detection System; Pierce, Rockford, IL).

\section{Chromatin immunoprecipitation (ChIP)}

BxPC3 cells were cross-linked with $1 \%$ formaldehyde for $10 \mathrm{~min}$ at $37^{\circ} \mathrm{C}$, then rinsed with cold PBS, harvested and lysed with 1\% SDS, $10 \mathrm{mM}$ EDTA, and $50 \mathrm{mM}$ Tris- $\mathrm{HCl}(\mathrm{pH}$ 8.1) containing a protease inhibitor cocktail (Roche, Indianapolis, IN). Chromatin was fragmented by two sequential sonications (2 min each) with $30 \mathrm{sec}$ on/off cycles and a Bioruptor sonicator (Diagenode, Denville, NJ) at its highest intensity. The soluble chromatin was diluted in buffer containing 1\% Triton, $2 \mathrm{mM}$ EDTA, $150 \mathrm{mM}$ $\mathrm{NaCl}$, and $20 \mathrm{mM}$ Tris- $\mathrm{HCl}(\mathrm{pH} 8.1)$ and added to Protein-G (Qiagen, Valencia, CA) pre-incubated with control IgG or anti-Flag antibody. After $24 \mathrm{~h}$, precipitates were washed 5 times and immune complexes were eluted using $100 \mathrm{ml}$ of $1 \%$ SDS and $0.1 \mathrm{M} \mathrm{NaHCO}$. The samples were incubated overnight at $65^{\circ} \mathrm{C}$ to reverse cross-linking, and the DNA was purified with a PCR purification kit (Qiagen, Valencia, CA).

\section{DNA Library preparation and high-throughput sequencing}

The purified DNA products were modified for Illumina Whole-Genome Chromatin IP sequencing using an Illumina Genomic DNA Sample Prep kit as follows: size-selected DNAs were end-repaired by T4 DNA polymerase and phosphorylated by T4 DNA polymerase and T4 polynucleotide kinase. The products were incubated with Klenow DNA Polymerase (Illumina) to generate $3^{\prime}$ adenine overhangs and then ligated to Illumina adapters, which contain 5' thymine overhangs. The adapter-ligated products were purified on QIAquick spin columns (Qiagen), PCR-amplified with Phusion DNA Polymerase (Finnzymes) for 30 cycles using an Illumina genomic DNA primer set. The PCR products were purified on QIAquick and MinElute columns (Qiagen).The quality of the DNA was assessed and quantified using an Agilent DNA 1000 Series II assay and NanoDrop ND-1000 spectrophotometer (Thermo Scientific) and the DNA was diluted to $10 \mathrm{nM}$. Cluster generation and sequencing were performed using a Standard Cluster Generation kit and a Cycle Solexa Sequencing kit on the Illumina Cluster Station and Illumina HiSeq2000 sequencer following the manufacturer's instructions. Sequencing was carried out by the Research \& Cooperation Division, BGI-Shenzhen.

\section{Bioinformatics}

Primary sequencing data consisted of: (i) Basic read maps; (ii) Peak region scans, including peak 
region detection, peak counts, average peak length, median peak length; (iii) The associated genes with sample peaks; (iv) The depth of coverage distribution of the mapped reads of the samples in the gene region; (v) GO function notability enrichment analysis of peak-related genes. SOAPaligner/soap2 was used for mapping sequencing reads to the genome. The SOAPaligner/soap2 is a program for faster and efficient alignment for short oligonucleotide onto reference sequences Genome Reference Consortium GRCh37/hg19 was used as reference genome. After filtering, and aligning read tags to reference sequences http://hgdownload.cse.ucsc.edu/ goldenPath/hg19/database/refGene.txt.gz), we calculated the average read coverage for all non-overlapping 50-bp slide windows of the genome. MACS 1.4.0 software was used for identifying potential peaks.

Kyoto Encyclopedia of Genes and Genomes (KEGG) pathway mapping is a procedure for mapping molecular datasets, especially large-scale datasets in genomics, transcriptomics, proteomics, and metabolomics, to the KEGG pathway maps for biological interpretation of higher-level systemic functions. Pathway annotations of the Notch1, Notch2 and CSL target genes were obtained from KEGG (http://www.genome.jp/kegg/). In the KEGG database, one gene may be involved in several pathways or interact with several other genes. Also, the KEGG Atlas website provides a mapping interface and allows mapping of genes as colored lines/circles in the global map. Pathway categories with a FDR $<0.01$ were marked.

\section{Whole-genome expression profiling}

The BxPC3 gene expression dataset was downloaded from GEO (accession number GSE15550). The dataset consists of microarray data, and the signal intensities were used as expression values.

\section{Statistical analyses}

All experiments were performed a minimum of three times. Data are means \pm SDs and were compared using Student's t-tests. A P-value $<0.05$ was considered to be statistically significant. Statistical analyses were performed with Office 2013 software (Microsoft) for Windows.

\section{Results}

\section{Notch 1, Notch2 and CSL Binding Sites on the BxPC3 cell genome}

As Notch 1 and Notch 2 have been implicated as important transcription factors in pancreatic cancer, and CSL is one of the downstream effectors for Notch family proteins, we mapped Notch1, Notch2 and CSL binding sites in the genome of $\mathrm{BxPC} 3$ pancreatic cancer cells. The quality of antibodies used for ChIP-Seq is a crucial factor that contributes to the quality of the data. Antibodies that offer high sensitivity and specificity are required for ChIP-Seq because they allow the detection of enrichment peaks without substantial background noise. It is also important to consider the potential cross-reactivity of antibodies with closely related protein family members. Because lack of specific CHIP-grade antibodies, FLAG-tagged CSL, Notch1 NICD and Notch2 NICD were expressed (Supplementary Fig S1), and anti-FLAG antibodies are used for the CHIP-Seq experiments.

MACS (Model-based Analysis of ChIP-Seq) is a command line tool designed to analyze data generated by ChIP-Seq experiments in eukaryote. MACS is used to identify transcription factor binding sites and histone modification enriched regions based on the ChIP-Seq data with or without controls. We used MACS 1.4.0 software and peak-calling algorithms to identify potential binding sites referred to as enriched regions. Analysis of CHIP-seq data identified 598, 745 and 85 statistically significant peaks of reads for Notch1, Notch2 and CSL, respectively. Only 49 of 598 Notch1 peaks (8.19\%) overlapped with CSL peaks, whereas 49 of the 98 CSL peaks $(57.65 \%)$ overlapped with Notch1 peaks (Fig. $3 \mathrm{~A})$. Only 39 of the 745 Notch2 (5.23\%) peaks overlapped with CSL peaks, whereas $45.88 \%$ of CSL peaks overlapped with Notch2 peaks (Fig. 3A). Thus, only small numbers of Notch1 and Notch2 binding sites overlap with those of CSL, indicating that most Notch1 and Notch2 functions are CSL-independent. Only $8.23 \%$ (49) of the Notch1 peaks overlapped with the Notch2 peaks, and $6.58 \%$ of the Notch2 peaks overlapped with the Notch1 peaks. We found that Notch1 and Notch2 have 48 and 43 target genes on chromosome 1, respectively, but they only share a common target, HCRTR1 (Fig. 3B; Supplementary Fig. S2). Other chromosomes have similar patterns, for example, on the chromosome 22, 32 and 17 target genes were found for Notch1 and Notch2, respectively, but only one gene (SMTN) was shared by Notch1 and Notch2 (Fig. 3C). Therefore Notch1 and Notch2 have different binding profiles in pancreatic cancer cells.

\section{Pathways relevant to Notch 1 , Notch 2 and CSL target genes}

We used the KEGG pathway mapping tool to identify the pathways biologically related to the ChIP-Seq-based Notch1, Notch2 and CSL target genes, consisting of the genes significantly enriched in 
each set. Table 1 shows the top 10 pathways associated with the Notch1, Notch2 and CSL target genes. The PI3K-AKT pathway is an intracellular signaling pathway important in regulating the cell cycle. Therefore, it is directly related to cellular quiescence and proliferation; if this pathway

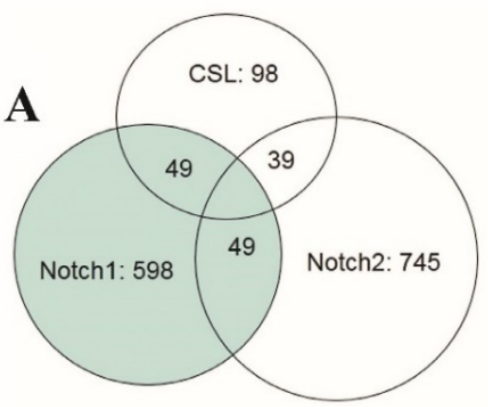

Notch1 and Notch2 target genes on chromosome 1

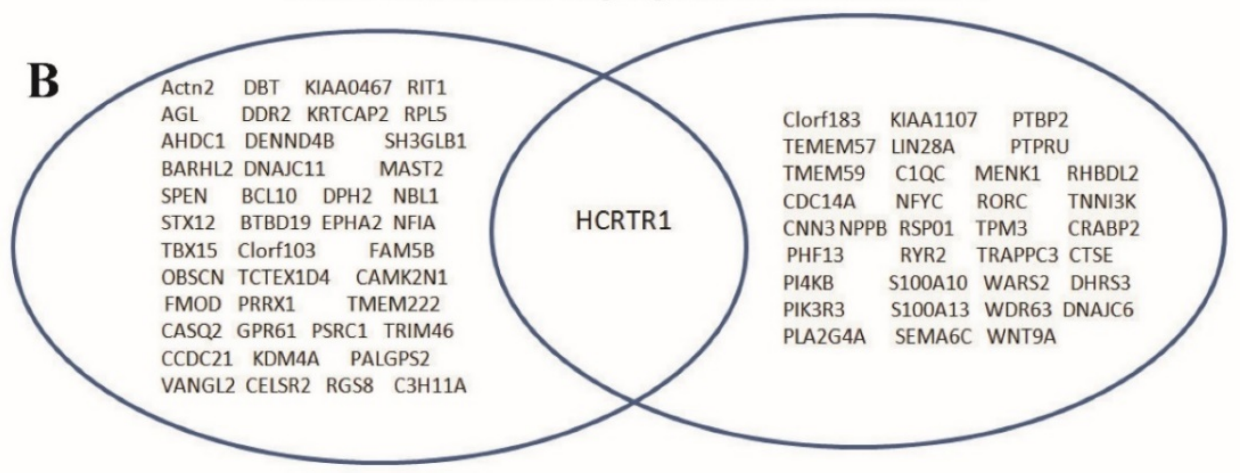

is overactive, it will reduce apoptosis and allow proliferation. We identified PI3K-AKT pathway as the most significant canonical pathway associated with the target genes of all three proteins, Notch1, Notch2 and CSL, thus supporting their roles in cell cycle control and cell growth.
Figure 3. Overlap of Notch1, Notch2 and CSL binding sites. Number of Notch1, Notch2 and CSL binding sites and overlapping binding sites are shown. (A) Analysis of CHIP-seq data identified 598, 745 and 85 statistically significant peaks of reads for Notch1, Notch2 and CSL. Number of Notch1, Notch2 and CSL binding sites and overlapping binding sites are shown. (B) Notch1 and Notch2 only share a few target genes. The figure shows Notch1 and Notch2 target genes on chromosome 1. Only one gene is targeted by both Notch1 and Notch2 on chromosome 1 . (C) Target gene distribution of CSL and Notch family proteins in chromosome 22 . Notch1 and Notch2 only share one gene (SMTN) on chromosome 22. The numbers are path coefficients.

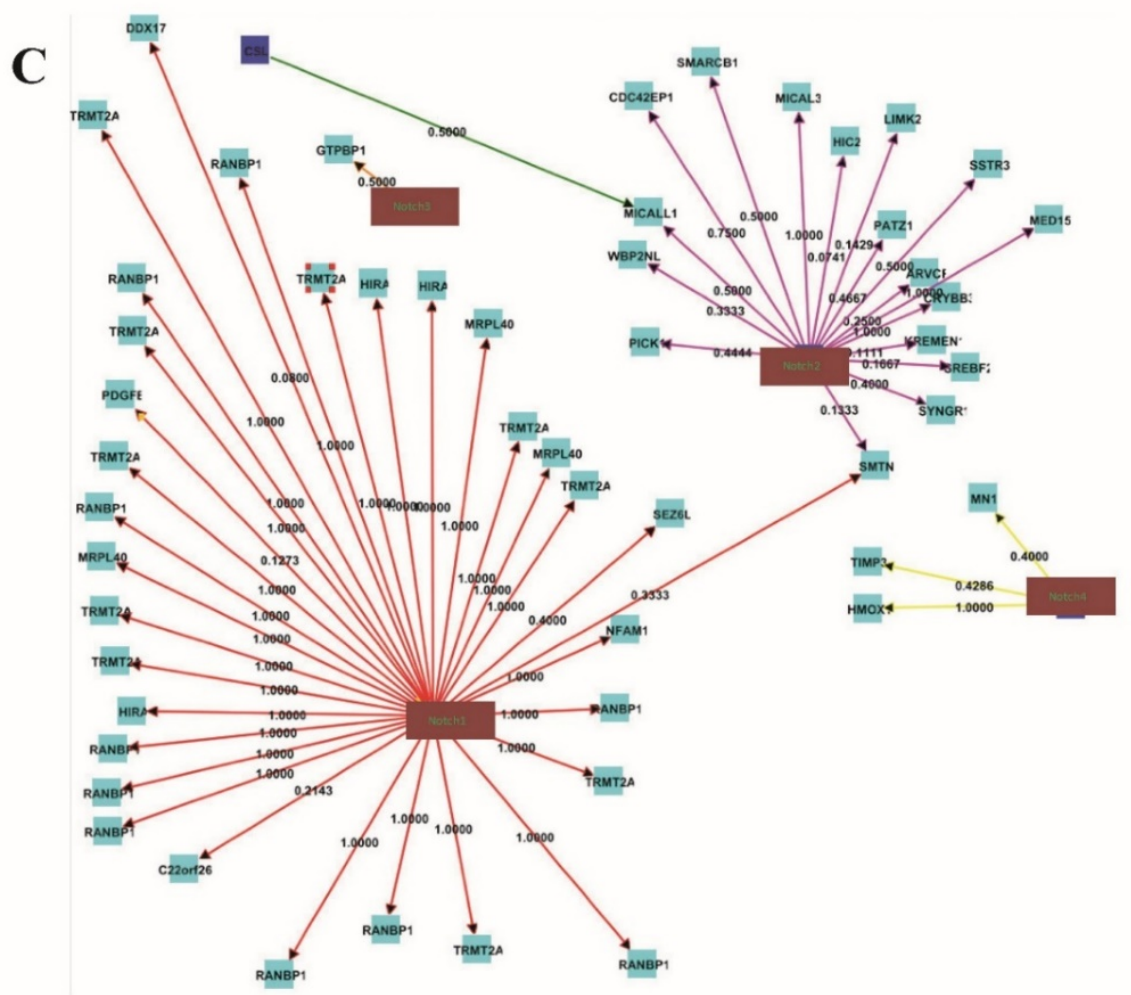


Besides the PI3K-AKT pathway, we identified transcriptional misregulation in cancer, metabolic pathways, the Ras pathway and the MAP kinase pathway as the most significant pathways linked to the Notch1 target genes. Pathways in cancer, transcriptional misregulation in cancer and viral carcinogenesis were associated with Notch2 target genes. Some Notch1, Notch2 and CSL target genes are associated with the PI3K-AKT pathway, the actin cytoskeleton and cancer microRNAs in cancer, but most of these target genes do not overlap. In the PI3K-AKT pathway, CSL only shares the target genes IL-4 and TSC1 with both Notch1 and Notch2, ITGB4 with Notch1, and CHRM1 with Notch2, but most of the target genes implicated in the same pathway are different. For example, Notch1 and Notch2 have 15 and 16 target genes, respectively, in the PI3K-AKT pathway, but only 3 are shared: CDKN1A, IL-4 and TSC1 (data not shown).

\section{Notch 1 target genes and cancer pathways}

The Notch1 target genes associated with cancer pathways are CCDC6, CDKN1A, FGFR2, GNB3, MET, MYC, PDGFB, PTCH1, RUNX1T1 (Table 2; Supplementary Fig. S3). In the gene expression dataset for BxPC3 in the NCBI GEO database (Dataset GSE15550), the average expression level is 433.66 for the all probes used. In that dataset, the Notch1 target genes associated with cancer pathways, namely genes MET, MYC, CCDC6 and CDKN1A, have expression levels exceeding this average level (i.e. >433.6). The gene CDKN1A codes for $\mathrm{p} 21$, which is a potent cyclin-dependent kinase inhibitor (CKI) [9]. CCDC6 encodes a coiled-coil domain-containing protein and may function as a tumor suppressor [10].

The KEGG Atlas website provides a mapping interface to allow mapping of genes as colored lines/circles in the global map. MET codes for the hepatocyte growth factor (HGF) receptor c-Met and has been implicated in driving proliferation and invasion, and is associated with a poor prognosis in pancreatic cancer [11]. c-Myc is a member of the MYC family of transcription factors [12]. MET and MYCs are thought to be oncogenes, and CCDC6 and CDKN1A tumor suppressor genes (TSGs). These results indicate that Notch1 targets both oncogenes and TSGs, and not all Notch1 target genes are highly expressed.

Table 1. Top 10 KEGG pathways relevant to CHIP-Seq-based Notch1, Notch2 and CSL target genes.

\begin{tabular}{|c|c|c|c|}
\hline \multicolumn{4}{|c|}{ Pathways (gene numbers) } \\
\hline Rank & Notch1 target genes & Notch2 target genes & CSL target genes \\
\hline 1 & PI3K-Akt signaling pathway (15) & PI3K-Akt signaling pathway (16) & Regulation of actin cytoskeleton (4) \\
\hline 2 & Transcriptional misregulation in cancer (10) & Pathways in cancer (13) & PI3K-Akt signaling pathway (4) \\
\hline 3 & Metabolic pathways (10) & Transcriptional misregulation in cancer (10) & cAMP signaling pathway (3) \\
\hline 4 & Pathways in cancer (9) & Viral carcinogenesis (9) & Tight junction (2) \\
\hline 5 & Ras signaling pathway (9) & cAMP signaling pathway (9) & $\begin{array}{l}\text { Arrhythmogenic right ventricular cardiomyopathy } \\
\text { (ARVC) (2) }\end{array}$ \\
\hline 6 & MAPK signaling pathway (9) & Metabolic pathways (9) & Calcium signaling pathway (2) \\
\hline 7 & Proteoglycans in cancer (9) & HTLV-I infection (9) & Jak-STAT signaling pathway (2) \\
\hline 8 & Regulation of actin cytoskeleton (8) & Neuroactive ligand-receptor interaction (9) & Hypertrophic cardiomyopathy (HCM) (2) \\
\hline 9 & HTLV-I infection (8) & Ras signaling pathway (8) & Herpes simplex infection (2) \\
\hline 10 & MicroRNAs in cancer (7) & Epstein-Barr virus infection (7) & MicroRNAs in cancer (2) \\
\hline
\end{tabular}

Notes: By importing Entrez Gene IDs of ChIP-Seq-based Notch1, Notch2 and CSL target genes into the Functional Annotation tool of KEGG, KEGG pathways showing significant relevance to the set of imported genes were identified.

Table 2. List and expression level of Notchl target genes in cancer pathway.

\begin{tabular}{|c|c|c|c|}
\hline Notch1 target genes & Full name of the gene & Illumina ID & Gene expression level \\
\hline MET & met proto-oncogene (hepatocyte growth factor receptor) & ILMN_12011 & 4048.37 \\
\hline MYC & v-myc myelocytomatosis viral oncogene homolog (avian) & ILMN_28130 & 3006.24 \\
\hline CCDC6 & coiled-coil domain containing 6 & ILMN_19206 & 2311.41 \\
\hline CDKN1A & cyclin-dependent kinase inhibitor 1A (p21, Cip1) & ILMN_5895 & 1260.56 \\
\hline GNB3 & guanine nucleotide binding protein ( $\mathrm{G}$ protein), beta polypeptide 3 & ILMN_4600 & 87.09 \\
\hline PDGFB & platelet-derived growth factor beta polypeptide (simian sarcoma viral (v-sis) oncogene homolog) & ILMN_18749 & 80.97 \\
\hline PTCH1 & patched homolog 1 (Drosophila) & ILMN_18640 & 76.33 \\
\hline FGFR2 & fibroblast growth factor receptor 2 & ILMN_20933 & 71.53 \\
\hline RUNX1T1 & runt-related transcription factor 1 ; translocated to, 1 (cyclin D-related) & ILMN_13370 & 67.37 \\
\hline
\end{tabular}


Table 3. List and expression level of Notch2 target genes in cancer pathway.

\begin{tabular}{|c|c|c|c|}
\hline Notch2 target genes & Full name of the gene & Ilumina ID & Gene expression \\
\hline TPM3 & tropomyosin 3 & ILMN_17262 & 11010.9 \\
\hline NF-кB IA & nuclear factor of kappa light polypeptide gene enhancer in B-cells inhibitor, alpha & ILMN_6745 & 10786.49 \\
\hline NF-кB 1 & nuclear factor of kappa light polypeptide gene enhancer in B-cells 1 & ILMN_26921 & 6345.35 \\
\hline CDKN1A & cyclin-dependent kinase inhibitor 1A (p21, Cip1) & ILMN_5895 & 1260.56 \\
\hline GSK3B & glycogen synthase kinase 3 beta & ILMN_7421 & 932.78 \\
\hline GNAQ & guanine nucleotide binding protein (G protein), q polypeptide & ILMN_18320 & 621.61 \\
\hline $\mathrm{BAX}$ & BCL2-associated $X$ protein & ILMN_10832 & 140 \\
\hline MDM2 & Mdm2 p53 binding protein homolog (mouse) & ILMN_21265 & 114.98 \\
\hline PGF & placental growth factor & ILMN_27436 & 90.5 \\
\hline FOXO1 & forkhead box $\mathrm{O} 1$ & ILMN_4600 & 87.09 \\
\hline RASGRP2 & RAS guanyl releasing protein 2 (calcium and DAG-regulated) & ILMN_10397 & 82.93 \\
\hline FN1 & fibronectin 1 & ILMN_4516 & 72.35 \\
\hline GNG3 & guanine nucleotide binding protein (G protein), gamma 3 & ILMN_7558 & 71.26 \\
\hline
\end{tabular}

\section{Notch2 target genes and cancer pathways}

Notch2 target genes associated with cancer pathway are TPM3, NF-кB IA, NF-кB 1, CDKN1A, GSK-3 $\beta$, GNAQ, BAX, MDM2, PGF, FOXO1, RASGRP2 (Table 3; Supplementary Fig. S4). TPM3 has the highest expression level among the genes in the gene expression dataset for BxPC3 in the NCBI GEO database, with a value of 11010.90 . The expression level of TPM3, NF-кB IA, NF-кB 1, CDKN1A, GSK-3 $\beta$, and GNAQ are also above the average, and the expression levels of BAX, MDM2, PGF, FOXO1 and RASGRP2 is lower than the average value. TPM3, NF-kB 1, GSK-3 $\beta$ and GNAQ are potential oncogenes and NF-KB IA and BAX are thought to be TSGs. These findings suggest that Notch2 targets both oncogenes and TSGs. Fig. 3C shows the Notch1 and Notch2 target different cancer-related pathways in the BxPC3 pancreatic cancer cells.

\section{Discussion}

The domains of Notch1 and Notch2 are highly conserved, and although both genes are expressed in pancreatic cancer, their functions (and differences in function) are not clear. Identification of their target genes may help to understand their roles in pancreatic cancer. CSL is a DNA binding transcription factor. In the canonical Notch signaling pathway, when ligands on neighboring cells interact with Notch receptors, Notch is proteolytically cleaved, and the notch receptor intracellular domain (NICD) translocates from the cell membrane to the nucleus where it forms a transcriptionally active ternary complex with CSL and a member of the Mastermind (MAM) family of coactivators. Assembly of the CSL-NICD-MAM ternary complex at a target gene is the switch for up-regulating transcription of that locus [13].

Our results indicate that most Notch1 and Notch2 target genes are CSL-independent. We found only a small subset of Notch1 and Notch2 peaks that overlapped with CSL peaks, indicating that most
Notch1 and Notch2 binding sites in the genome are not dependent on CSL. Factors other than CSL may be involved in Notch1 and Notch2 binding to the genome. Since about half of the CSL peaks overlap with Notch1 or Notch2 peaks, the functions of CSL appear to be more closely related to those of Notch1 and Notch2, than those of Notch1 and Notch2 are related to CSL. In addition, a few of some other co-factors may also be involved in CSL's activities. For example, the bHLH transcription factor Ptfla was found to be a co-factor of CSL and forms transcription complexes that can activate CSL target gene transcription [14].

Our results indicate that the profiles of the major pathways in which Notch1, Notch2 and CSL target genes are different. Moreover different Notch1, Notch2 and CSL target genes are associated with a given common pathway. For example, Notch1, Notch2 and CSL only share a few target genes in the PI3K-AKT pathway, namely IL-4, TSC1 and CDKN1A, while most of the other target genes do not overlap. This results support the notion that Notch1, Notch2 and CSL tend to play different roles even in the same pathway. Further studies are needed to identify the roles of Notch1, Notch2 and CSL in regulating the activity of a pathway that they have in common: do they play parallel, opposing or synergistic roles?

We found that Notch1 and Notch2 both bind to the same target gene CDKN1A in the cancer pathway. Gene CDKN1A codes for p21(CIP1/WAF1), which binds to and inhibits the activity of cyclin-CDK2, -CDK1, and -CDK4/6 complexes, and thus functions as a regulator of cell cycle progression in $G_{1}$ and $S$ phases. In addition to growth arrest, p21 can mediate cellular senescence. The expression of this gene is tightly controlled by tumor suppressor protein p53, through which p21 mediates p53-dependent cell cycle arrest in $G_{1}$ in response to a variety of stress stimuli [9]. When DNA damage or inhibitory extracellular 
signals are present, p53 increases and stimulates transcription of the $\mathrm{p} 21$ gene. As CDKN1A is a target of both Notch1 and Notch2, further study is required to establish the different roles of Notch1 and Notch2 in regulation of p21 expression.

The main Notch1 target genes are MET, MYC and CCDC6. HGF and its receptor, c-Met, have been implicated in driving proliferation, invasion, and a poor prognosis in pancreatic cancer [11]. In one report, HGF and MET mRNAs were measured in 59 primary pancreatic cancers and 51 normal samples, and the results showed that both factors are highly expressed in pancreatic cancer. This suggests that the HGF/c-Met axis plays an important role in the progression of pancreatic cancer and that targeting c-Met may be of therapeutic value [11]. Mounting evidence suggests that c-Myc participates in most aspects of cellular function, including metabolism, growth, differentiation, apoptosis, adhesion, and migration [12]. Amplification and/or over-expression of c-Myc are frequently detected in cancers including pancreatic cancer [12].

Besides CDKN1A, Notch1 also targets the potential tumor suppressor gene CCDC6, which encodes a coiled-coil domain-containing protein that is involved in DNA damage repair. When thyroid cancer cells were treated with etoposide or ionizing radiation (IR), CCDC6 underwent ATM-mediated phosphorylation at Thr 434, stabilizing nuclear CCDC6, indicating that this protein is involved in the response to ATM-mediated DNA damage. Impairment of CCDC6 gene function may have a role in thyroid carcinogenesis [13].

The Notch2 target genes in the cancer pathway are TPM3, NF-кBIA, NF-кB1, GSK-3 $\beta$ and GNAQ. TPM3 gene encodes a member of the tropomyosin family of actin-binding proteins and is a potential oncogene. TPM3 was overexpressed in human hepatocellular carcinoma and there was a potential link to epithelial-mesenchymal transition [14]. Also, TPM3 overexpression is associated with high-grade gliomas and increased mortality. The role of TPM3 in pancreatic cancer deserves further investigation.

It is interesting that Notch 2 targets both NF- $\mathrm{kB}$ and the NF-kB inhibitor, NF-kBIA, and both genes are highly expressed in BxPC3 pancreatic cancer cells. NF-kB plays a key role in proliferation, cell survival, and invasion by pancreatic cancer cells. It is constitutively activated in pancreatic cancer where its inhibition enhances the sensitivity of cancer cells to chemotherapeutic agents and death receptor-mediated apoptosis. NF-kBIA, also known as IKBa, is a member of the family of cellular proteins that inhibit the activity of NF-KB by masking its nuclear localization signal (NLS) and keeping it sequestered in an inactive state in the cytoplasm [15]. It may be a tumor suppressor [16]. Notch2 is potentially a key regulator of NF-kB and NF-kBIA expression. Further experiments are needed to identify additional factors involved in the regulation of NF-kB and NF-kBIA expression by Notch2. Obviously the NF-KB /NF-kBIA ratio is important for the activity of NF-KB signaling.

GSK-3 $\beta$ is also a potential Notch2 target gene. GSK-3 $\beta$ is a serine/threonine protein kinase that regulates multiple signaling pathways. Although its role in cancer is still debated, the overall results so far indicate that aberrant expression and activity of GSK-3 $\beta$ is a fundamental characteristic of a broad spectrum of cancers [17]. The potential involvement of GSK-3 $\beta$ in the invasiveness of pancreatic cancers and their resistance to gemcitabine and ionizing radiation, the two major obstacles to more effective treatment, have also been reported [18].

The role of GNAQ in pancreatic cancer has not been examined. GNAQ encodes the alpha subunit of the q class of heterotrimeric GTP binding protein $(\mathrm{Gq})$ that transduces signals from G-protein-coupled receptors (GPCRs), and stimulates $\beta$ phospholipase $C$ (PLC $\beta)$, which catalyzes the hydrolysis of phosphatidylinositol biphosphate (PIP2). Frequent somatic mutations were found in GNAQ in a subset of melanocytic neoplasms that did not have alterations in RAS or BRAF [19]. Genetic, biochemical and biological analysis has indicated that GNAQ is a bona fide human oncogene. The role of GNAQ in pancreatic cancer remains to be defined.

Notch2 may be involved in the regulation of the p53 pathway. Mdm2 is an important negative regulator of p53 and is a Notch2 target gene. Mdm2 functions both as an E3 ubiquitin ligase that recognizes the $\mathrm{N}$-terminal trans-activation domain (TAD) of p53 and an inhibitor of p53 transcriptional activation [20]. It has been reported that single-nucleotide polymorphisms are associated with the risk of cancer [21-23]. Detection of mutations and genomic polymorphisms in the Notch target sequences may provide valuable information about differences among individuals in terms of the Notch activity and help us to understand how the Notch pathways are involved in the risk of pancreatic cancer. Chen et al. reported that 8 genetic variants are predictive of early onset pancreatic cancer, and all of the 8 SNPs were predicted to play functional roles in the disruption of transcription factor and/or enhancer binding sites and most of them were expression quantitative trait loci (eQTL) of the target genes [24].

The Notch family protein-related gene regulation network is complex. As Notch1 and Notch2 target both oncogenes and TSGs, it is hard to 
define them as oncogenes or TSGs. Nevertheless, as they have different target gene profiles they may play distinct roles in pancreatic cancer.

\section{Supplementary Material}

Supplementary figures.

http://www.jcancer.org/v08p0065s1.pdf

\section{Acknowledgments}

This work was supported by The National Nature Science Foundation of China (grant no. 81372156) and a grant from the Major State Basic Research Development Program (2010CB529400).

\section{Competing Interests}

The authors have declared that no competing interest exists.

\section{References}

1. Bray SJ. Notch signaling: a simple pathway becomes complex. Nat Rev Mol Cell Biol. 2006 Sep;7(9):678-89.

2. Lobry C, Oh P, Aifantis I. Oncogenic and tumor suppressor functions of Notch in cancer: it's NOTCH what you think. J Exp Med. 2011 Sep 26;208(10):1931-5.

3. Mullendore ME, Koorstra JB, Li YM, et al. Ligand-dependent Notch signaling is involved in tumor initiation and tumor maintenance in pancreatic cancer. Clin Cancer Res. 2009 Apr 1;15(7):2291-301.

4. Andersson ER, Sandberg R, Lendahl U. Notch signaling: simplicity in design, versatility in function. Development. 2011 Sep;138(17):3593-612.

5. Fan X, Mikolaenko I, Elhassan I, et al. Notch1 and notch2 have opposite effects on embryonal brain tumor growth. Cancer Res. 2004 Nov 1;64(21):7787-93.

6. Miyamoto Y, Maitra A, Ghosh B, et al. Notch mediates TGF alpha-induced changes in epithelial differentiation during pancreatic tumorigenesis. Cancer Cell. 2003 Jun;3(6):565-76.

7. Mazur PK, Einwächter H, Lee M, et al. Notch2 is required forprogression of pancreatic intraepithelial neoplasia and development of pancreatic ductal adenocarcinoma. Proc Natl Acad Sci U S A. 2010 Jul 27;107(30):13438-43.

8. Kopan R, Ilagan MX. The canonical Notch signaling pathway: unfolding the activation mechanism. Cell. 2009 Apr 17;137(2):216-33.

9. Grabliauskaite K, Hehl AB, Seleznik GM, et al. p21(WAF1) (/Cip1) limits senescence and acinar-to-ductal metaplasia formation during pancreatitis. J Pathol. 2015 Feb;235(3):502-14.

10. Inuzuka H, Guggino G, Monaco R, et al. FBXW7 and USP7 regulate CCDC6 turnover during the cell cycle and affect cancer drugs susceptibility in NSCLC. Oncotarget. 2015 May 20;6(14):12697-709..

11. Jin $\mathrm{H}$, Yang $\mathrm{R}$, Zheng $\mathrm{Z}$, et al. MetMAb the one-armed 5D5 anti-c-Met antibody, inhibits orthotopic pancreatic tumor growth and improves survival. Cancer Res. 2008 Jun 1;68(11):4360-8.

12. Morra F, Luise C, Merolla F, et al. Therapeutic effects of an anti-Myc drug on mouse pancreatic cancer. J Natl Cancer Inst. 2014 Oct 11;106(12).

13. Merolla F, Pentimalli F, Pacelli R, et al. Involvement of H4(D10S170) protein in ATM-dependent response to DNA damage. Oncogene. 2007 Sep 13;26(42):6167-75.

14. Choi HS, Yim SH, Xu HD, et al. Tropomyosin3 overexpression and a potential link to epithelial-mesenchymaltransition in human hepatocellular carcinoma. BMC Cancer. 2010 Apr 1;10:122.

15. Carbone C, Melisi D. NF-kB as a target for pancreatic cancer therapy. Expert Opin Ther Targets. 2012 Apr;16 Suppl 2:S1-10.

16. Prigent $\mathrm{M}$, Barlat $\mathrm{I}$, Langen $\mathrm{H}$, et al. IkappaBalpha and IkappaBalpha/NF-kappa B complex are retained in the cytoplasm through interaction with a novel partner, RasGAP SH3-binding protein 2. J Biol Chem. 2000 Nov 17;275(46):36441-9.

17. Wilson W 3rd, Baldwin AS. Maintenance of constitutive IkappaB kinase activity by glycogen synthase kinase-3alpha/beta in pancreatic cancer. Cancer Res. 2008 Oct 1;68(19):8156-63.

18. Shimasaki T, Ishigaki $Y$, Nakamura $Y$, et al. Glycogen synthase kinase $3 \beta$ inhibition sensitizes pancreatic cancer cells to gemcitabine. J Gastroenterol. 2012 Mar;47(3):321-33.

19. Lamba S, Felicioni L, Buttitta F, et al. Mutational profile of GNAQQ209 in human tumors. PLoS One. 2009 Aug 31;4(8):e6833.

20. Duffy MJ, Synnott NC, McGowan PM, et al. p53 as a target for the treatment of cancer. Cancer Treat Rev. 2014 Dec;40(10):1153-60.

21. Liu M, Kurosaki T, Suzuki M, Enomoto $Y$, Nishimatsu H, Arai T, Sawabe M, Hosoi T, Homma Y, Kitamura T. Significance of common variants on human chromosome 8q24 in relation to the risk of prostate cancer in native Japanese men. BMC Genet. 2009 Jul 14;10:37.

22. Liu M, Suzuki M, Arai T, Sawabe M, Enomoto Y, Nishimatsu H, Kume H, Homma Y, Kitamura T. A replication study examining three common single-nucleotide polymorphisms and the risk of prostate cancer in a Japanese population. Prostate. 2011 Jul;71(10):1023-32

23. Suzuki M, Liu M, Kurosaki T, Suzuki M, Arai T, Sawabe M, Kasuya Y, Kato M, Fujimura T, Fukuhara H, Enomoto Y, Nishimatsu H, Ishikawa A, Kume H, Homma Y, Kitamura T. Association of rs6983561 polymorphism at 8q24 with prostate cancer mortality in a Japanese population. Clin Genitourin Cancer. 2011 Sep;9(1):46-52.

24. Chen J, Wu X, Huang Y, Chen W, Brand RE, Killary AM, Sen S, Frazier ML. Identification of genetic variants predictive of early onset pancreatic cancer through a population science analysis of functional genomic datasets. Oncotarget. 2016 Jul 29. doi: 10.18632/oncotarget.10924. [Epub ahead of print] PubMed PMID: 27486767. 